\title{
AUTOPERCEPCIÓN DE ENFERMEDAD PERIODONTAL EN ESCOLARES DE 12 AÑOS DE EDAD. CUENCA-ECUADOR
}

\section{Self-perception of Periodontal Disease in 12 years-old Schoolchildren. Cuenca-Ecuador.}

\author{
Alvear-Ordóñez Mónica Lizbeth ${ }^{1}$,Naula-Vicuña Carlos $^{2}$, Toral-Aguilera Diego Xavier ${ }^{3}$,Ledesma-Herrera \\ Kimberlyn Viviana ${ }^{4 *}$ \\ ${ }^{1}$ Odontóloga de la Carrera de Odontología de la Universidad Católica de Cuenca, Cuenca Ecuador \\ ${ }^{2}$ Especialista en Periodoncia- Docente de la Universidad Católica de Cuenca. \\ ${ }^{3}$ Especialista en Periodoncia de la Universidad de Chile. \\ ${ }^{4}$ Odontóloga de la Carrera de Odontología de la Universidad Católica de Cuenca, Cuenca Ecuador \\ *kimylh.95@gmail.com
}

\begin{abstract}
Resumen
Objetivo: Determinar la prevalencia de enfermedad periodontal (EP) autopercibida en escolares de 12 años de la parroquia urbana Gil Rámírez Dávalos- Cuenca- 2016. Materiales y Métodos: Se realizó una investigación de tipo descriptiva y retrospectiva. El tamaño de la muestra fue de 161 escolares de 12 años, a quienes se les aplicó un cuestionario de autovaloración del estado periodontal, diseñado y validado por los Centros de Control y Prevención de Enfermedades (CDC). Resultados: La prevalencia global de EP autopercibida fue de $81 \%$. No se pudieron comprobar diferencias en relación con este valor en función del sexo de los escolares ni tampoco en función del tipo de gestión escolar (pública, privada o mixta). Conclusión: La gran mayoría de los escolares encuestados manifestó percibir algún signo o síntoma de enfermedad periodontal. A través de este trabajo pionero pretendemos abrir el paso a nuevos estudios, con el fin de fomentar la importancia del diagnóstico temprano y la prevención de EP, así como promover la Salud Periodontal.
\end{abstract}

Palabras clave: Prevalencia, enfermedades periodontales, Autoreporte, Salud del Adolescente..

\begin{abstract}
Aim: To determine the prevalence periodontal disease (PD), through the use of a self-report instrument, in 12-year-old schoolchildren of the urban parish Gil Rámírez Dávalos- Cuenca- 2016. Materials and Methods: The study was descriptive and retrospective. The sample size was 161 schoolchildren of 12 years, who underwent a periodontal self-evaluation questionnaire, designed and validated by the Centers for Disease Control and Prevention (CDC). Results: The overall prevalence of self-reported PD was $81 \%$. We did not find any significant difference in this parameter when comparing children of both, nor when comparing children attending public, private or mixed institutions. Conclusion: The vast majority of schoolchildren surveyed reported perceiving some signs or symptoms of periodontal disease. Through this pioneering work we intend to open the way to new studies, in order to promote the importance of early diagnosis and prevention of PD, as well as promoting periodontal health.
\end{abstract}

Key words: Prevalence, Periodontal diseases, Self-report- Adolescent Health..

\section{INTRODUCCIÓN}

Las Enfermedades Periodontales (EP) se definen como un grupo de manifestaciones clínicas de origen infeccioso e inflamatorio, generalmente causadas por un mal control de placa bacteriana, que afectan a los tejidos de soporte de las piezas dentales. ${ }^{1}$ La falta de un correcto diagnóstico y un apropiado tratamiento influye en la progresión de estas enfermedades hacia estados severos, pudiendo causar incluso la pérdida de piezas dentales. ${ }^{2,3}$ Según la nueva clasificación propuesta en el 2017 por la American Academy of Periodontology (AAP) y la European Federation of Periodontology (EFP), las EP se clasifican en tres grandes grupos: 1.Salud Periodontal-Gingivitis- Condiciones Gingivales, 2. Periodontitis y 3. Otras Manifestaciones que afectan al Periodonto (Manifestaciones por Enfermedades Sistémicas-Desarrollo y Condiciones Adquiridas). ${ }^{4}$ 
Desde hace algún tiempo las EP se han convertido en un problema de salud pública, ya que por su etiología microbiana están relacionadas con varias condiciones sistémicas como, por ejemplo: la diabetes, enfermedad cardiovascular, artritis reumatoide, obesidad y síndrome metabólico, entre otras. ${ }^{5,6}$ Datos epidemiológicos recientes confirman que estas enfermedades afectan entre el $40 \%$ y $50 \%$ de la población mundial y, junto con la caries dental, son las afecciones orales con mayor prevalencia en el mundo. ${ }^{7,8}$ Pese a ello, la población en general desconoce la importancia de las periodontopatías, e ignora que pueden padecer de esta condición, dejando de acudir a los servicios de Salud Pública y retrasando así su tratamiento.

En países con altos ingresos como EEUU, la prevalencia de las EP alcanza hasta un $60 \%$ en la población escolar. ${ }^{9}$ Según la Organización Panamericana de la Salud, en países latinoamericanos como Perú, Ecuador y Colombia esta prevalencia aumenta hasta un $85 \%$ de la población en general. En México, la prevalencia de las EP en escolares es del $44 \%$, de ahí radica la importancia de obtener datos que nos proporcionen información que explique cómo la EP está afectando en la calidad de vida de la población..$^{9-11}$ En los últimos tiempos se han realizado estudios que se han evidenciado una incidencia significativa de las EP en niños y adolescentes, pudiendo estar asociada a los cambios hormonales que ocurren durante la pubertad. ${ }^{9}$ Un estudio realizado por Bimstein y Matsson en niños de 5 a 15 años de edad reveló que existe una mayor prevalencia y niveles más altos de placa e inflamación gingival en niños de 8 y 12 años. ${ }^{12}$ Por estas razones la Organización Mundial de la Salud (OMS) considera la edad de 12 años relevante para este tipo de estudios, por ser una edad de comparación internacional para nuevas investigaciones. ${ }^{13}$

La autopercepción es una forma de estimar el estado de salud y está influenciada por la función física, la presencia de enfermedades y las limitaciones funcionales. Se trata de evaluar cómo el paciente se siente, piensa o percibe su estado de salud. La autopercepción del estado de salud oral se puede valorar mediante el llamado autoreporte o "Self Report", que busca evaluar la necesidad de atención odontológica percibida por el paciente. Por supuesto, se trata de información subjetiva y para obtenerla algunos autores han diseñado cuestionarios que evalúan los problemas funcionales, sociales y psicológicos relacionados con problemas de salud bucodental. ${ }^{14}$

Según Miller, la simplicidad y el bajo costo del uso de este tipo de cuestionarios de autopercepción de molestias causadas por EP los convierte en herramientas muy útiles para la vigilancia de la salud oral y los estados de salud periodontal en las poblaciones, sin olvidar que el diagnóstico clínico es, sin duda, más certero y superior. ${ }^{15}$ Sin embargo, también es factible sospechar la existencia de la enfermedad a través del impacto que la misma tiene sobre la calidad de vida de las personas. A diferencia del examen clínico, esta estrategia exige menos tiempo y recursos a utilizar, puesto que no requiere de examinadores especializados y permite la obtención de una gran cantidad de información sobre la población en una sola instancia. ${ }^{16-19}$

En vista de que no existen suficientes investigaciones epidemiológicas acerca de cómo perciben su salud periodontal escolares de la Sierra Ecuatoriana, el presente estudio tuvo como objetivo determinar en qué medida escolares de 12 años de la parroquia Urbana Gil Ramírez Dávalos, CuencaEcuador auto percibían la enfermedad periodontal, durante el año -2016.

\section{MATERIALES Y MÉTODOS}

Se realizó un estudio descriptivo, observacional y retrospectivo. Se incluyeron en el estudio escolares de 12 años inscritos en las escuelas públicas-particulares y fiscomisionales de la Parroquia Urbana Gil Ramírez Dávalos del Cantón Cuenca en el año 2016, que presentaron sus respectivos consentimientos informados firmados por el representante legal. Se excluyeron del estudio escolares que padecían alguna enfermedad sistémica, síndromes, o alguna discapacidad mental o física.

El instrumento empleado para el estudio fue el Auto Reporte de Periodoncia, diseñado y validado por los Centers for Disease Control and Prevention de EEUU (CDC), con la colaboración de la American Academy of Periodontology (AAP) para diagnosticar la enfermedad periodontal autopercibida, ${ }^{20}$ El cuestionario constaba de 8 preguntas, las cuales permitían a los escolares valorar su estado periodontal de manera subjetiva; el estudio fue aprobado por el Comité de Bioética de la Universidad Católica de Cuenca.

Para el cálculo de la prevalencia, se consideraron "enfermos" aquellos escolares que respondieron positivamente a 3 o más preguntas del cuestionario. Los resultados se analizaron mediante estadística descriptiva, basada en distribuciones relativas y absolutas de los parámetros. Para evaluar la posible relación entre la autopercepción de EP y la gestión de los establecimientos educativos, se clasificaron los establecimientos según la gestión educativa como unidades educativas públicas (Fiscales), privadas (Particulares), y mixtas (Fiscomisionales).

\section{RESULTADOS}

La muestra estudiada, estuvo constituida por 161 escolares de 12 años de edad, de los cuales 114 eran de sexo femenino y 47 de sexo masculino, es decir el $71 \%$ de los escolares eran mujeres y $29 \%$ varones. 
La gran mayoría de los escolares encuestados (81\%) manifestó percibir algún signo o síntoma de enfermedad periodontal, mientras que solo el $19 \%$ no percibieron la EP como tal. Al evaluar la autopercepción de EP, la prevalencia alcanzó $82 \%$, en el caso de las niñas y $83 \%$ en el caso de los niños, por lo cual no hay diferencias significativas entre escolares de ambos sexos

Las diferencias de autopercepción en función del tipo de establecimiento educativo (público, privado o mixto) no fueron importantes (Tabla 1). En efecto, mientras que en las escuelas privadas (particulares) o públicas (fiscales) el porcentaje de escolares que autopercibieron las EP fue de 80$81 \%$, en las escuelas fiscomisionales este valor alcanzó el $83 \%$.

De las preguntas incluidas en el cuestionario, dos de ellas fueron respondidas afirmativamente en un porcentaje elevado de los casos. En efecto, el $48 \%$ de los escolares respondió que sus encías sangraban, mientras que el $53 \%$ afirmó notar que sus dientes no se veían bien. Finalmente, entre el 24 y el $25 \%$ de los escolares afirmaron sentir que tenían mal aliento o que probablemente sufrían de algún tipo de enfermedad de la cavidad bucal.

\section{DISCUSIÓN}

El resultado más importante que refleja el presente estudio es que 4 de cada 5 estudiantes de 12 años percibieron la enfermedad periodontal, de una u otra manera, mientras que el $19 \%$ de escolares no la percibieron. Es decir, la gran mayoría de los escolares encuestados manifestaron sentir o percibir alguna afección en sus tejidos periodontales.

El presente estudio evaluó la prevalencia de las enfermedades periodontales de manera indirecta, es decir, mediante la aplicación de un cuestionario de autopercepción. Se trata de una forma sencilla y rápida de obtener información útil con fines de intervención en materia de salud oral. Esta estrategia permite, además, motivar a la población para explorar sus necesidades de tratamiento a nivel oral y dar a conocer las afecciones periodontales, particularmente en relación a cómo pueden afectar en la calidad de vida de las personas.

El uso de un cuestionario de autopercepción de EP tiene una ventaja adicional: aumentar la demanda en los servicios de salud pública, permitiendo así el diagnóstico en estadios prematuros de la enfermedad, evitando su progresión hacia formas más graves. ${ }^{5,21,22}$ Esta estrategia de promoción de la salud oral pretende que los individuos acepten que padecen una condición patológica, para motivarlos a buscar un servicio de salud para tratar sus afecciones a tiempo. ${ }^{23}$ Se trata de una idea que ya fue discutida por Sheiham, quien propuso este instrumento como una alternativa sencilla para planificar los servicios de salud sobre la base de una autovaloración de la necesidad de tratamientos periodontales.

Las investigaciones sobre la autopercepción de las EP son escasas a nivel regional, y no se han reportado estudios de este tipo en escolares de 12 años en la región andina, hasta el momento. Allí radica, precisamente, la importancia de este estudio ya que existe una gran necesidad de promover la prevención de las EP en adolescentes, por formar parte de un grupo etario susceptible. En efecto, estudios realizados en países Latinoamericanos en grupos de adolescentes revelaron altas prevalencias de la EP. Por ejemplo, en el 2009, Doncel y Vidal reportaron un $82 \%$ de prevalencia de enfermedad periodontal en pacientes adolescentes de la Habana. ${ }^{24}$ En otro estudio similar, realizado en la ciudad de Santiago de Chile por Pareja y Gracia el mismo año, se reveló que un $60 \%$ de pacientes, entre los 7 y 14 años, padecía inflamación gingival. ${ }^{9}$ Por su parte, en un estudio realizado con escolares de 4 a 14 años, en la ciudad de León (México) en el 2013, Rocha confirmó que la prevalencia de enfermedad periodontal incrementaba según la edad. Finalmente, en un estudio realizado en el 2008 en Ciudad de México, Juárez y col. reportaron una prevalencia del $70 \%$ de EP en adolescentes. ${ }^{11,25}$ Todo lo anterior ratifica la importancia de tratar la inflamación gingival en estadios tempranos, particularmente en este grupo de pacientes.

En un estudio de autoreporte publicado por Pérez en el 2011, el 47,7\% de los adolescentes encuestados manifestaron que les sangraban las encías, el $16 \%$ tenían movilidad dental y el $90 \%$ no usaba hilo dental. ${ }^{7}$ En el presente estudio los resultados fueron similares (al $48 \%$ le sangraban las encías, y el $14 \%$ sentían al menos un diente flojo. ${ }^{13,26}$

El cuestionario de autopercepción de EP tiene algunas ventajas adicionales, entre las que podemos destacar las siguientes: se puede obtener la información de la condición periodontal, de una manera más rápida, simple y a bajo costo; no requiere de examinadores especializados, ni de instrumentos esterilizados (como ocurre con la evaluación clínica). De hecho, este tipo de autoreportes fue utilizado por Khader, quien empleó la información obtenida para el control de las EP. ${ }^{27}$ Según Miller, ${ }^{15}$ el cuestionario estaba orientado originalmente hacia el control y prevención de la enfermedad periodontal, razón por la cual fue aprobado por el Grupo de Vigilancia de Enfermedades Periodontales. Sin embargo, el mismo autor manifiesta que las preguntas fueron diseñadas para ser respondidas por encuestados que tenían poco conocimiento de las enfermedades gingivales, con el fin de evidenciar si los pacientes percibían que tenían algún problema en su salud.

En el 2013 Ramos hizo énfasis en la necesidad de validar un instrumento adecuado para diagnosticar las EP 
Tabla 1. Auto reporte de enfermedad periodontal según la Gestión Educativa

\begin{tabular}{lcccccc} 
& \multicolumn{2}{c}{ PARTICULAR } & \multicolumn{2}{c}{ FISCOMISIONAL } & \multicolumn{2}{c}{ FISCAL } \\
& $\mathrm{N}$ & $\%$ & $\mathrm{~N}$ & $\%$ & $\mathrm{~N}$ & $\%$ \\
PERCIBE & 129 & 80 & 133 & 83 & 130 & 81 \\
NO PERCIBE & 32 & 20 & 27 & 17 & 30 & 19 \\
\hline
\end{tabular}

\section{Encuesta de Autopercepción Periodontal}

1. ¿Piensa Ud. Que tal vez sufra de enfermedad de las encías?

2. ¿ Alguna vez le han hecho cirugía de las encias?

3. . ¿ Alguna vez le han hecho raspado o alisado en las raíces?

4. ¿Alguna vez se le aflojado un diente?

5. ¿Alguna vez le han dicho un dentista que ha perdido hueso alrededor de los dientes?

\section{6. ¿ Le sangran las encías?}

7. Siente ud. Ole han dicho que tiene mal aliento?

8. ¿Ha notado que alguno de sus dientes no parece verse bien?

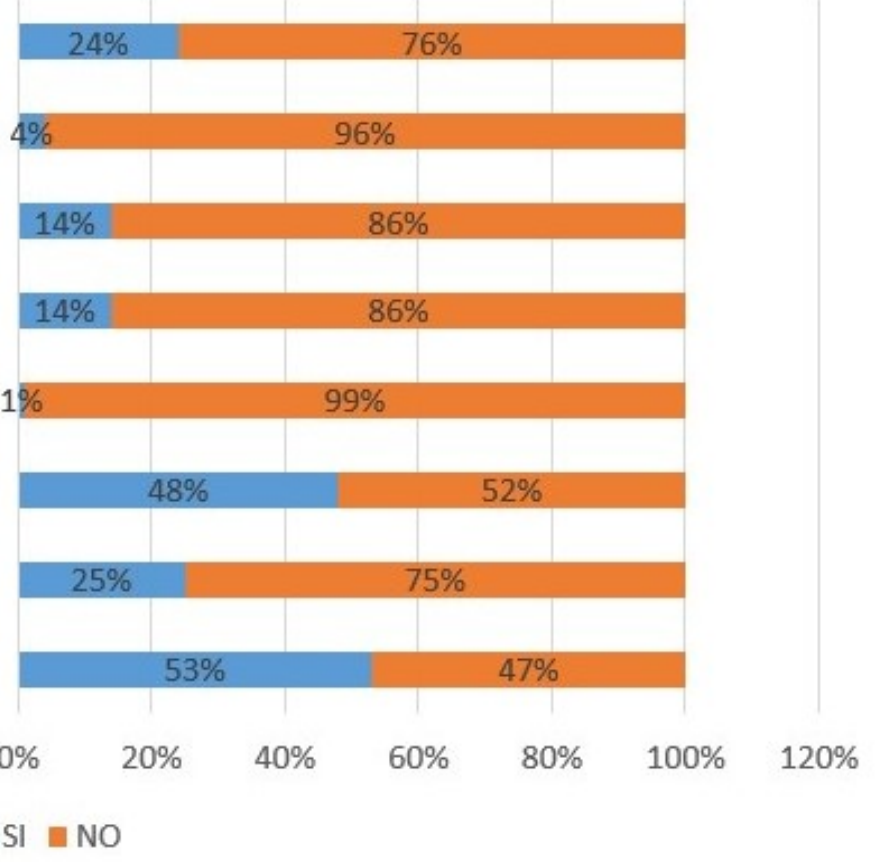

Figura 1. Encuesta de Autopercepción de Enfermedad Periodontal

a través de la autopercepción de la salud bucal, pues los resultados podrían variar en función de factores tales como el nivel socioeconómico de los escolares y la asistencia previa al odontólogo. ${ }^{16}$ Por ello, nuestro estudio agrupó a los escolares según el tipo de gestión educativa del colegio al que acudían; sin embargo, las diferencias observadas no fueron importantes. En relación con este aspecto destacamos que, el análisis de las preguntas incluidas en el cuestionario, nos permitió comprobar que una de ellas (¿has notado que alguno de tus dientes no se ve bien?) fue respondida afirmativamente en más de la mitad de los casos. Sin embargo, es de resaltar que esta pregunta no evalúa específicamente la $\mathrm{EP}$, ya que se podría sospechar de cualquier patología de la cavidad oral.

Una de las limitaciones de nuestro estudio es no haber podido comparar los resultados de prevalencia de EP estimada mediante autoreporte versus la prevalencia calculada en base al estudio clínico. Esta comparación habría permitido tener una evaluación más clara del valor del instrumento empleado como herramienta diagnóstica. No obstante, se trata de un estudio que aborda una problemática, frecuentemente subestimada, y que podría permitir el diagnóstico temprano de la enfermedad periodontal, mejorando así las condiciones de salud bucal de los pacientes y su calidad de vida.

\section{CONCLUSIONES}

El $81 \%$ de los escolares encuestados manifestaron percibir algún tipo de problema periodontal. El cuestionario de auto reporte se puede utilizar como una herramienta de apoyo al diagnóstico de las EP. No hubo diferencias significativas en cuanto a la autopercepción de las EP auto al comparar individuos de diferente sexo. Tampoco pudimos 
demostrar diferencias en relación a la autopercepción 11 Juárez-López MLA, Murrieta-Pruneda JF, TeodosioProcode las EP, al comparar escolares que acuden a colegios administrados bajo diferentes tipos de gestión educativa. pio E. Prevalencia y factores de riesgo asociados a enfermedad periodontal en preescolares de la Ciudad de México. Gaceta médica de México. 2005;141(3):185-189.

CONFLICTO DE INTERESES: Los autores no 12 Clerehugh V, Kindelan S. Guidelines for periodontal screemanifiestan ningún conflicto de interés. ning and management of children and adolescents under 18 years of age. British Society of Periodontology and the

AGRADECIMIENTOS: Los autores agradecen al British Society of Pediatric Dentistry. 2012;p. 1-25.

Dr. Luis Andrés Yarzábal (Departamento de Investigación, 13 Lagos Lagos A, Juárez Membreño IA, Iglesias Gómez Carrera de Odontología. Universidad Católica de Cuenca) por su apoyo en la revisión crítica y la edición de este manuscrito. M. Necesidad de tratamiento periodontal e higiene oral en adolescentes de 12 años de Llanquihue. Revista clínica de periodoncia, implantología y rehabilitación oral. 2014;7(2):72-75.

\section{Referencias Bibliográficas}

1 Sociedad Española de Periodoncia y Osteointegración. Manual SEPA de Periodoncia y Terapéutica de Implantes: Fundamentos y guía práctica. Ed. Médica Panamericana; 2005.

2 Lorenzo S, Piccardo V, Alvarez F, Massa F, Alvarez R. Enfermedad periodontal en la población joven y adulta uruguaya del interior del país.Relevamiento Nacional 20102011. Odontoestomatología. 2013;15(SPE):35-46.

3 Pulido-Rozo M, Gonzalez-Martínez F, Rivas-Muñoz F. Enfermedad periodontal e indicadores de higiene bucal en estudiantes de secundaria Cartagena, Colombia. Revista de Salud Pública. 2011;13:844-852.

4 Caton J, Armitage G, Berglundh T, et al. A new classification scheme for periodontal and peri-implant diseases and conditions - Introduction and key changes from the 1999 classification. J Periodontol. 2018;89(Suppl 1):S1-S8.

5 Carvajal P. Enfermedades periodontales como un problema de salud pública: el desafío del nivel primario de atención en salud. Revista clínica de periodoncia, implantología y rehabilitación oral. 2016;9(2):177-183.

6 Bracho R, Ruiz M, Bohórquez D, Pérez, Ligia, Rodríguez, Esperanza, Rivera, Luis, Bermúdez, Valmore, Higiene Oral y Enfermedad Periodontal en Individuos con Síndrome Metabólico. Revista Latinoamericana de Hipertensión.2011;6(4).

7 Orta RP, Huerta HAS, Zavala AC. Prevalencia y severidad de enfermedad periodontal crónica en adolescentes y adultos. Oral. 2011;12(39):799-804.

8 Medina A, Arbeláez M, Guzmán I. Perfil microbiológico subgingival de pacientes con periodontitis crónica en una población de Colombia. Avances en periodoncia e implantología oral. 2012;24(1):47-53.

9 Vásquez MP. Prevalencia y necesidad de tratamiento periodontal en escolares de la institución educativa Andrés Bello. KIRU Revista de la Facultad de Odontología-Universidad de San Martín de Porres. 2015;6(1).

10 Research S, Committee T. Position paper: diagnosis of periodontal diseases. Journal of periodontology. 2003;74(8):1237-1247.

14 Duque A, Cuartas C, Muñoz C, Salazar C, Sánchez Y. Nivel de conocimiento sobre enfermedad periodontal en una muestra de empleados en Medellín. Revista CES Odontología. 2011;24(2):43-47.v

15 Miller K, Eke PI, Schoua-Glusberg A. Cognitive evaluation of self-report questions for surveillance of periodontitis. Journal of periodontology. 2007;78:1455-1462.

16 Ramos RQ, Bastos JL, Peres MA. Diagnostic validity of self-reported oral health outcomes in population surveys: literature review. Revista Brasileira de Epidemiologia. 2013;16:716-728.

17 Blicher B, Joshipura K, Eke P. Validation of self-reported periodontal disease: a systematic review. Journal of dental research. 2005;84(10):881-890.

18 Kini V, Patil RU, Pathak T, Prakash A, Gupta B, etal. Diagnosis and management of periodontal disease in children and adolescents: A brief review. Journal of Dental and Allied Sciences. 2016;5(2):78.

19 Vered Y, Sgan-Cohen HD. Self-perceived and clinically diagnosed dental and periodontal health status among young adults and their implications for epidemiological surveys. BMC Oral Health. 2003;3(1):3.

20 ChattopadhyayA. Oral health epidemiology :principlesand practice. Jones \& Bartlett Publishers; 2010.

21 Fernández González C, Núñez Franz L, Díaz Sanzana N. Determinantes de salud oral en población de 12 años. Revista clínica de periodoncia, implantología y rehabilitación oral. 2011;4(3):117-121.

22 Popoola BO, Dosumu EB, Ifesanya JU. Periodontal status and treatment need among adolescents in Ibadan, Southwestern Nigeria. Brazilian Journal of Oral Sciences. 2015;14(2):117-121.

23 Sheiham A. Oral health, general health and quality of life. Bull World Health Organ. 2005 Sep; 83(9): 644

24 Doncel Pérez C, Vidal Lima M, del Valle Portilla MdC. Relación entre la higiene bucal y la gingivitis en jóvenes. Revista Cubana de medicina militar. 2011;40(1):40-47.

25 25. Murrieta-Pruneda JF, Juárez-López LA, Linares-Vieyra C, Zurita-Murillo V, Meléndez-Ocampo AF, Ávila-Martínez $\mathrm{CR}$, et al. Prevalencia de gingivitis asociada a la higiene oral, ingreso familiar y tiempo transcurrido desde la última 
consulta dental, en un grupo de adolescentes de Iztapalapa, Ciudad de México. Boletín médico del Hospital Infantil de México. 2008;65(5):367-375.

26 Lindhe J, Karring T, Lang NP. Periodontologia clinica e implantologia odontologica/Clinical Periodontology and Implant Dentistry. vol. 1. Ed. Médica Panamericana; 2009.

27 Khader Y, Alhabashneh R, Alhersh F. Development and validation of a self-reported periodontal disease measure among Jordanians. International dental journal. 2015;65(4):203-210.

Recibido: 10 de enero de 2019.

Aceptado: 30 de abril de 2019. 